\title{
Vitamina D e COVID-19: Uma revisão integrativa
}

\author{
Vitamin D and COVID-19: An integrative review \\ Vitamina D y COVID-19: Una revisión integradora
}

Recebido: 09/07/2021 | Revisado: 18/07/2021 | Aceito: 22/07/2021 | Publicado: 29/07/2021

\author{
Jordy Silva de Carvalho \\ ORCID: https://orcid.org/0000-0002-9276-8927 \\ Universidade Católica de Pernambuco, Brasil \\ E-mail: jordycarvalho@hotmail.com \\ Marina Galdino da Rocha Pitta \\ ORCID: https://orcid.org/0000-0002-4219-2683 \\ Universidade Federal de Pernambuco, Brasil \\ E-mail: marinagaldinopitta@gmail.com \\ Ítalo Rufino de Queiroz Fernandes \\ ORCID: https://orcid.org/0000-0001-8017-8068 \\ Universidade de Rio Verde, Brasil \\ E-mail: italorufinof@gmail.com \\ Trinnye Luizze Santos \\ ORCID: https://orcid.org/0000-0002-5537-7492 \\ Faculdade Nova Esperança de Mossoró, Brasil \\ E-mail: santosluizze@gmail.com \\ Luzilene Pereira de Lima \\ ORCID: https://orcid.org/0000-0003-1038-2711 \\ Universidade Católica de Pernambuco, Brasil \\ E-mail: luzilene.1011@gmail.com \\ Rhuann Pontes dos Santos Silva \\ ORCID: https://orcid.org/0000-0002-2126-6655 \\ Universidade Católica de Pernambuco, Brasil \\ E-mail: rhuannpontes02@gmail.com \\ Manuela Barbosa Rodrigues de Souza \\ ORCID: https://orcid.org/0000-0002-7773-100X \\ Universidade Católica de Pernambuco, Brasil \\ E-mail: manu.brsouza@gmail.com
}

\section{Resumo}

O presente artigo tem como objetivo investigar a influência da vitamina D associada no tratamento de casos graves da COVID-19. Diversos estudos convergem para a importância de um organismo com um bom sistema imunológico para que não haja risco de progressão da doença, ressaltando, portanto, a importância da presença de vitamina D no corpo humano. Nessa revisão, iremos discutir as mais recentes evidências sobre o metabolismo e mecanismo de ação da vitamina D no corpo, seus alvos biológicos, sua importância no papel imunológico, seus benefícios para a saúde, e sua relação com a patogenicidade da COVID-19, particularmente em casos severos. Apesar da grande quantidade de material científico relacionado ao tema, o resultado do presente artigo evidencia que ainda faltam investigações que correlacionam perfis genéticos e respostas virais, além de estudos clínicos randomizados que avaliem a influência da suplementação com vitamina D no controle e progressão do quadro clínico da COVID-19.

Palavras-chave: Coronavírus; Colecalciferol; COVID-19; Sistema imunológico.

\begin{abstract}
The objective of this article is to investigate the influence of associating vitamin D in the treatment of severe cases of COVID-19. Several studies match on the importance of having a good immune system to avoid the risk of disease progression, enhancing the importance of having vitamin D in the human body. In this review, we will discuss the latest evidence on the metabolism and mechanism of action of vitamin D in the immune system, its biological targets, its importance in the immunological role, its health benefits, and its relationship with the pathogenicity of COVID-19, particularly in severe cases. However the large quantity of scientific data related to the topic, the result of this review shows that there is still having a lack of investigations that correlate genetic data and viral responses, in addition to randomized clinical studies that appraises the influence of vitamin D supplementation on the control and progression of the clinical condition of COVID-19.
\end{abstract}

Keywords: Coronavirus; Cholecalciferol; COVID-19; Immune system. 


\section{Resumen}

El objetivo de este artículo es investigar la influencia de la asociación de vitamina $\mathrm{D}$ en el tratamiento de casos graves de COVID-19. Varios estudios coinciden en la importancia de tener un buen sistema inmunológico para evitar el riesgo de progresión de la enfermedad, destacando la importancia de tener vitamina D en el cuerpo humano. En esta revisión, discutiremos las últimas evidencias sobre el metabolismo y mecanismo de acción de la vitamina $\mathrm{D}$ en el sistema inmunológico, sus dianas biológicas, su importancia en el papel inmunológico, sus beneficios para la salud y su relación con la patogenicidad del COVID-19., particularmente en casos severos. Sin embargo, ante la gran cantidad de datos científicos relacionados con el tema, el resultado de esta revisión muestra que aún faltan investigaciones que correlacionen datos genéticos y respuestas virales, además de estudios clínicos aleatorizados que evalúen la influencia de la suplementación con vitamina D en el control y progresión de la condición clínica de COVID-19.

Palabras clave: Coronavirus; Colecalciferol; COVID-19; Sistema inmune.

\section{Introduçãa}

Há mais de um ano começaram a surgir relatos da SARS-CoV-2, em que a doença passou a ser chamada coronavírus (COVID-19), ela causa sintomas semelhantes aos da pneumonia. Em 5 de janeiro de 2020, foi relatado que esse vírus infectou 59 pessoas na cidade de Wuhan, na província chinesa de Hubei. Após quinze dias, as autoridades chinesas haviam relatado mais de 200 infecções e 3 mortes. No final de janeiro, a Organização Mundial da Saúde (OMS) declarou uma Emergência de Saúde Pública de Preocupação Internacional (“Editorial Nature”, 2021).

Apesar da intensa busca por potenciais produtos naturais e sintetizados para o tratamento de pacientes com COVID-19, nenhum medicamento anti-SARS-CoV-2 específico se mostrou eficaz no combate desse vírus causador da pneumonia infecciosa aguda. Por outro lado, muitos estudos forneceram uma compreensão profunda dos efeitos terapêuticos da medicina convencional e tradicional, dos fatores imunológicos envolvidos e dos mecanismos de ação relacionados ao vírus SARS-CoV-2 (Bourhia et al., 2020).

A vitamina D é um composto lipossolúvel essencial e um pró-hormônio esteróide. Foi demonstrado que a deficiência deste micronutriente, ou sua insuficiência, está associada a doenças cardiovasculares, tumores, infecções respiratórias, asma e doenças infectocontagiosas (Teixeira et al., 2018). Um estudo recente mostrou que o nível plasmático médio de 25 hidroxivitamina $\mathrm{D}$ foi significativamente mais baixo entre as pessoas com teste positivo do que negativo para COVID-19 (Merzon et al., 2020).

Desta forma, a deficiência de vitamina D pode desempenhar um papel no desenvolvimento da doença em estudo, interferindo diretamente na replicação viral e atuando de forma imunomoduladora e anti-inflamatória. O presente trabalho busca avaliar e revisar de que forma ocorre a modulação, expressão e secreção da 25-hidroxivitamina D em pacientes portadores da COVID-19, visto que o interferon tipo 1, quimiocinas e citocinas pró-inflamatórias foram associados em pacientes com o referido vírus e hipovitaminose (Liu et al., 2020).

\section{Metodologia}

Trata-se de uma Revisão de Literatura Integrativa, com suporte teórico para sua importância descrita por Koche, J.C.(2011). Foi realizada mediante coleta nas bases de dados eletrônicas do MedLine/PubMED ((Biblioteca Nacional de Medicina do Instituto Nacional de Saúde dos Estados Unidos) e LILACS (Literatura Latino-americana e do Caribe em Ciências da Saúde), entre os anos de 2011 e 2021, utilizando-se os descritores indexados no DECs//MESH (Descritores em Ciências da Saúde) 'COVID-19”, '’Vitamin D” e 'Immunology”, realizando-se a seguinte associação: (COVID-19) OR (Coronavirus) AND (Vitamin D) AND (Immune System). Foram encontrados 112 artigos ao todo, sendo 54 selecionados, analisados e discutidos, tendo em vista que correspondiam à informações pertinentes para o tema em questão.

Dentre os critérios de inclusão utilizados, encontram-se artigos e periódicos disponíveis na íntegra e completos, nos idiomas inglês e espanhol, com pesquisa de abordagem para o estudo de testes controlados e aleatórios, séries de casos, meta- 
análises e ensaios clínicos. Como critérios de exclusão, foram estabelecidos que artigos que não versavam à temática ou ao período temporal delimitado, ou que possuíam desfecho e conclusão inadequados ou inconsistentes foram desconsiderados. Duplicatas também foram excluídas. Terapêuticas. Após a revisão, foram estabelecidos pontos de concordância dos estudos no que diz respeito a vitamina D e sua relação com o COVID -19, abordando-se os aspectos fisiopatológicos, epidemiológicos e terapêuticos dessa.

\section{Resultados e Discussão}

\subsection{Vitamina D: metabolismo e mecanismos por meio de COVID-19}

Cerca de $80 \%$ da vitamina D no corpo humano é sintetizada endogenamente, enquanto o restante vem da dieta, possuindo uma estrutura molecular muito semelhante (Figura 1), com a diferença de apenas uma dupla ligação (destacada em azul). Ergocalciferol difere de colecalciferol por apenas uma dupla ligação entre os carbonos 22 e 23 e, além disso, por um grupo metil no carbono 24. Ambas as vitaminas são sintetizadas por fotólise através da exposição solar. Após a ingestão do ergocalciferol e do colecalciferol, ambos seguem a via metabólica no fígado para originar a 25-hidroxivitamina D (Castro et al., 2011).

Figura 1. Estrutura molecular do ergocalciferol e colecalciferol.

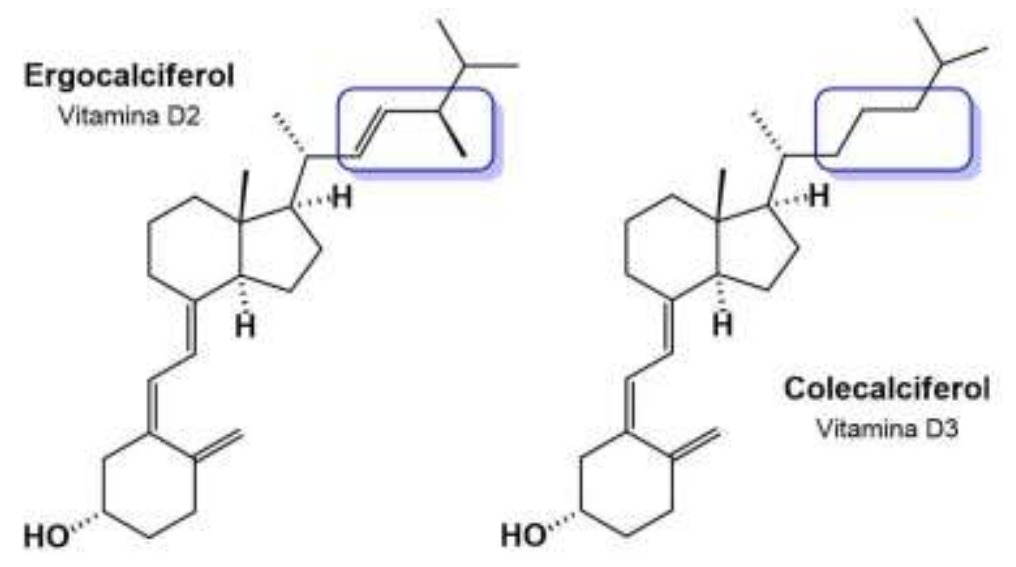

Fonte: Autores (2021).

Tais precursores são carreados no fígado por uma proteína de ligação à vitamina D (DBP). A vitamina D é hidroxilada no carbono 25 pela hidroxilase codificada pelo gene CYP2R1. A 25-hidroxivitamina D é a principal forma sérica de vitamina D e foi considerada um precursor na forma ativa $1,25(\mathrm{OH})_{2} \mathrm{D}$, porém, em elevadas concentrações, possui a capacidade de ligar-se ao receptor da vitamina D (VDR). Ademais, várias células extra renais expressam megalina, especialmente as células da paratireóide, osteoblastos e a $1 \alpha$-hidroxilase. Por isso, a produção local de $1,25(\mathrm{OH})_{2} \mathrm{D}$ ocorre em diversos tecidos (Guillaume, 2017).

As vitaminas D2 e D3 sofrem hidroxilação pelo citocromo P450 e irão dar origem à 25-hidroxivitamina D, que é a forma majoritária na circulação, a hidroxilação, bem como a formação da forma da vitamina D encontrada em maior parte na circulação ocorrem no fígado. No sangue, cerca de 85 a $90 \%$ desta vitamina está conectada à proteína ligadora de vitamina D (VDBP), 10 a 15\% se encontra ligada à albumina e o restante, menos de 1\%, circula na forma livre. Poucos estudos têm demonstrado a biodisponibilidade da vitamina D ligada à albumina, assim, a expressão "biodisponibilidade da vitamina D” é empregada para a forma da 25-hidroxivitamina D que não está ligada à VDBP (Bikle, 2017).

Alguns modelos experimentais marcados com ausência do receptor VDR possibilitam uma melhor compreensão da 
atividade tissular específica. Por exemplo, a falta de VDR leva a um aumento da massa ventricular, elevação dos níveis de peptídeo natriurético tipo B (brain natriuretic peptide, BNP) e desregulação das metaloproteinases cardíacas e dos fibroblastos, promovendo uma matriz extracelular fibrótica e levando à dilatação ventricular e desacoplamento eletromecânico (Al Mheid \& Quyymi, 2017). Após a ligação da vitamina D ao VDR, o complexo formado promove a ativação ou a supressão gênica, com o auxílio de proteínas reguladoras. Em contrapartida, o VDR apresenta também respostas rápidas não genômicas pela indução de canais de cálcio voltagem-dependentes, ocasionando em um aumento do influxo celular de cálcio e estimulação de outros mensageiros, como o AMP cíclico, a proteína quinase A e a fosfolipase C (Castro, 2011).

A natureza virulenta do coronavírus torna necessária uma identificação rápida e eficaz de agentes terapêuticos promissores. Alguns dos alvos descritos na literatura são: proteína não estrutural 15 (NSP15), enzima conversora de angiotensina 2 (ACE2), macrófagos de resposta imune, células supressoras derivadas de mielóide (MDSCs), dentre outros (Castro, 2011).

A NSP15, codificada pelo coronavírus, desempenha um papel vital no ciclo de vida, nos processos de replicação e transcrição, bem como na virulência do mesmo, que desempenha um papel indispensável no processamento de RNA e evasão imune viral do sistema imunológico do hospedeiro (Rameez et al., 2020). A NSP15 é um antagonista do interferon (IFN) e suprime a produção de interferon- $\beta$ (INF- $\beta$ ) por meio da atividade de endoribonuclease (Shalayel et al., 2020).

Shalayel et al. investigou a afinidade de ligação potencial da vitamina D com os locais de ligação putativos da endoribonuclease NSP15 da COVID-19. Eles encontraram três sítios de ligação prospectivos com os ligantes, sendo que quando comparada com Remdesivir, Cloroquina e Hidroxicloroquina, a vitamina D apresentou a maior potência com a mais forte interação em termos de menor energia de ligação (LBE), menor raiz média do desvio quadrático (RMSD) e menor intensidade de inibição Ki do que os outros compostos padrão.

Outros compostos também foram investigados para a proteína NSP15. Visando o estudo de reaproveitamento de drogas, Khan et al., em 2020, pesquisou esta proteína não estrutural contra uma biblioteca interna de 123 compostos antivirais obtidas no banco de dados DrugBank. Dois fármacos candidatos, Simeprevir e Paritaprevir, alcançaram as energias livres de ligação baixas para NSP15 $(-259,522 \pm 17,579$ e $-154,051 \pm 33,628 \mathrm{~kJ} / \mathrm{mol}$, respectivamente) e podem ser potenciais agentes terapêuticos contra SARS-CoV-2 (Khan et al., 2020).

Os INFs são um grupo de proteínas de sinalização necessárias para a defesa antiviral. Liberados por células infectadas por vírus ou leucócitos, eles medeiam uma variedade de respostas imunológicas inatas e adaptativas por meio da regulação positiva de mais de 300 genes estimulados por interferon (ISGs)14. Os IFNs do tipo I são divididos em vários subtipos, incluindo $-\alpha,-\beta,-\omega,-\varepsilon,-\tau,-\delta$ e $-\kappa$, cada um com funções independentes (Newmark et al., 2017). Gauzzi \& Fantuzzi, em 2020, propuseram que a vitamina D pode cooperar com IFNs tipo I para controlar a fase inicial da infecção por SARS-CoV-2, esses interferon são os mediadores naturais mais poderosos da atividade antiviral em humanos, e há evidências crescentes de que uma resposta fraca ou retardada do IFN do tipo I contribui para a gravidade do COVID-19. Alguns trabalhos suportam a evidência da importância da vitamina D nas infecções virais, levantando a hipótese de que um nível adequado de vitamina D no momento da infecção ajuda a resposta protetora contra IFN tipo I precoce, e reforça a imunidade antiviral inata ao SARS-CoV-2. No entanto, são necessários mais estudos para compreender melhor a ligação entre o status de vitamina D, a resposta de IFN tipo I e COVID-19 (Gauzzi \& Fantuzzi, 2020).

Estudos estruturais das sequências da proteína receptora do vírus revelaram que o SARS-CoV-2 pode reconhecer a enzima conversora de angiotensina 2 (ECA2) de humanos e outras espécies animais, como furões, gatos e outros como hospedeiros intermediários. Este receptor auxilia na transmissão do vírus entre humanos e entre espécies. ECA2 é uma metalopeptidase de zinco que é um antagonista da enzima de conversão da angiotensina (ECA). O principal papel da ECA2 é converter angiotensina II em angiotensina-(1-7) e promover a via relevante por meio de seus efeitos anti-inflamatórios e antioxidantes em alguns casos (Mardani et al., 2020). 
Há evidências epidemiológicas e clínicas mostrando que a vitamina D pode reduzir as lesões pulmonares por vários mecanismos, incluindo a indução de peptídeos antimicrobianos, reduzindo as concentrações de citocinas pró-inflamatórias, em particular por meio de seus efeitos inibitórios na ativação da renina-angiotensina sistema (RAS), e aumentando as citocinas antiinflamatórias. Também é provável que a vitamina D possa exercer efeitos protetores contra a COVID-19 por meio da supressão da resposta das citocinas e reduzir o risco de síndrome do desconforto respiratório agudo (SDRA). No entanto, o envolvimento do ECA2 deu origem a sugestões conflitantes sobre a contribuição do ECA2 no tratamento do COVID-19 (Bertoldi et al., 2020; Mardani et al., 2020).

Mardani et al., em 2020, encontraram alterações significativas nos níveis de vitamina D e ECA, bem como razão neutrófilos/linfócitos (NLR) no grupo de pacientes COVID-19. A concentração sérica de ECA mostrou um aumento significativo no grupo de pacientes e foi significativamente maior nos indivíduos falecidos. Observando os indivíduos com diferentes níveis de vitamina D, a concentração da ECA apresentou maior quantidade entre os indivíduos com concentração insuficiente de vitamina $\mathrm{D}$ em relação ao grupo controle ( $\mathrm{p}<0,0001$ ). No entanto, os resultados desse estudo não encontraram ligação importante entre as concentrações de vitamina D no sangue com o risco de COVID-19, nem sugeriram a utilidade da medição de vitamina D na prática clínica para avaliar o risco de infecção por COVID-19 (Mardani et al., 2017). Mais estudos precisam ser feitos para a obtenção de dados mais concretos.

Os receptores de vitamina D se apresentam nos macrófagos, células dendríticas e em outras células do sistema imune. Por consequência, muitos dos genes-alvo possuem funções ligadas à resposta imunológica (Kongsbak et al., 2013). Assim, eles conduzem a transcrição de vários genes diferentes, incluindo os que codificam peptídeos antimicrobianos. Dois desses alvos são a catelicidina e a defensina, que atuam na diminuição da taxa de replicação do vírus e proporcionam a quimiotaxia de macrófagos e de outras células do sistema imune para os órgãos que estão inflamados (Fiske et al., 2019).

Diante do exposto, a suplementação de vitamina $\mathrm{D}$ pode oferecer benefícios quando se trata de possibilitar a diminuição da resposta hiperinflamatória que há, principalmente, nos pulmões de pacientes com COVID-19. Ademais, a presença de vírus respiratórios comuns pode limitar o crescimento do vírus SARS-CoV-2, e como as crianças contraem essas infecções mais que os adultos, elas têm estatisticamente mais chances de serem protegidas por essa reação (Nickbakhsh et al., 2019; Kloc et al., 2021).

\subsection{O papel da vit. D no sistema imunológico}

A vitamina D tem como função mais conhecida a homeostase do cálcio, atuando, por conseguinte, na manutenção da integridade óssea, tendo também um papel importante ao promover a maturação das células do sistema imunológico (Alexander et al., 2020). Diante disso, se destaca a íntima relação no funcionamento das células T (Kratz et al., 2018).

O gene CYP27B1, expresso nas células T, é responsável por processar a 25-hidroxivitamina D (pré-hormônio da vitamina D) em calcidiol (hormônio ativo). O calcidiol segue para os rins, onde o calcitriol é formado e, quando há a ligação do calcitriol às células T, estas células do sistema imune serão capazes de cumprir as suas funções (Sousa, 2016).

Há uma inversa associação entre os níveis circulantes do biomarcador de vitamina D, 25(OH)-D3 e biomarcadores inflamatórios, como a Proteína C Reativa (PCR) (Liu et al., 2014). Corroborando com este achado, destaca-se que pessoas com ótimos níveis de vitamina D são mais saudáveis (Lichtenstein et al., 2013).

Foi indicada uma relação entre a vitamina D e a COVID-19 quando esta teve menor taxa de infecção em localidades que tinham níveis mais altos do micronutriente entre os indivíduos e/ou latitudes mais baixas (Ilie et al., 2020; Rhodes et al., 2020). Vale salientar que um estudo realizado com pacientes da atenção primária e de clínicas especializadas demonstrou a associação entre a hipovitaminose com um maior número de admissão hospitalar, bem como gravidade da doença e óbito (Mendy et al., 2020). 
Carpagnano et al., 2021 demonstrou que a taxa de mortalidade de pacientes com COVID-19 hospitalizados na Unidade de Terapia Respiratória Intermediária (UTIR) da Policlínica de Bari de 11 de março a 30 de abril de 2020 foi de 5\%, para aqueles que tinham um nível suficiente de vitamina $\mathrm{D}$ (25-hidroxivitamina $\mathrm{D}$ sérica $\geq 30 \mathrm{ng} / \mathrm{mL}$ ). Já em pacientes que possuíam grave deficiência dessa vitamina (25-hidroxivitamina $\mathrm{D}$ sérica $\leq 10 \mathrm{ng} / \mathrm{mL}$ ) tiveram uma taxa de mortalidade de 50\% depois de 10 dias internados (Carpagno et al., 2021).

Idade mais avançada, tabagismo, diabetes, hipertensão e obesidade são fatores de risco para deficiência de vitamina D (Siuka et al., 2020). Para o combate dessa deficiência é importante uma dieta com a presença de alimentos como: peixes, leite fortificado, cogumelos e ovos. Além disso, ela pode ter origem sob a pele através da exposição a luz ultravioleta, luz solar ou se necessário através de suplementos (Lichtenstein et al., 2013).

Nesta perspectiva, a deficiência de vitamina $\mathrm{D}$ é um fator relevante para que haja uma má progressão do quadro viral (Haan et al., 2014). Desse modo, a terapia vitamínica adjuvante pode ter potencial terapêutico para pacientes com COVID-19 (Bae \& Kim, 2020).

\subsection{Benefícios da vitamina D para a COVID-19}

A vitamina D também é chamada de colecalciferol (vitamina D3) ou ergocalciferol (vitamina D2), o termo engloba um grupo de moléculas secosteroides derivadas do 7-dehidrocolesterol interligadas através de uma cascata de reações fotolíticas e enzimáticas que acontecem em células de diferentes tecidos. Sua via biossintética começa com a radiação UVB de 7 dehidrocolesterol na pele nua exposta à luz solar, que é a fonte primária. A vitamina D3, além de atuar como reguladora da fisiologia osteomineral, também está envolvida na homeostase de outros processos celulares, como a síntese de interleucinas inflamatórias, e participa da regulação dos processos de multiplicação e diferenciação celular (Martineau \& Forouhni, 2020).

Vários estudos vêm apontando que tal nutriente inibe a proliferação de linfócitos, a produção de anticorpos e a síntese de citocinas por meio de monócitos e estimulação imunológica mediada por células. Sendo assim, o seu baixo nível está relacionado ao aumento de doenças infecciosas e não infecciosas, principalmente as infecções do trato respiratório superior. Além disso, os metabólitos da vitamina $\mathrm{D}$ apoiam os mecanismos antivirais efetores inatos, incluindo a indução de peptídeos antimicrobianos e autofagia. Por isso, acredita-se que também haja a modulação favorável às respostas do hospedeiro e à síndrome respiratória aguda grave do coronavírus, SARS-CoV-2, tanto na fase viral inicial quanto na fase hiperinflamatória tardia da COVID-19 (Liu et al., 2021).

Existem vários fatores que podem influenciar para que a vitamina D possa ser utilizada no tratamento do novo coronavírus, dentre eles sua capacidade de reduzir a imunidade celular inata e estimular as tempestades de citocinas, que estão inteiramente relacionadas ao agravamento da síndrome do desconforto respiratória agudo associado à COVID-19. Além disso, a vitamina $\mathrm{D}$ suporta os peptídeos antimicrobianos produzidos no epitélio do trato respiratório, o que torna as infecções virais e os sintomas do coronavírus improváveis, e por fim, ajuda na diminuição da resposta inflamatória à infecção por SARS-CoV-2 (Liu et al., 2021).

\subsection{COVID-19 \& mortalidade}

Epidemiologicamente, o espectro de mortalidade da população idosa infectada com COVID-19 está diretamente relacionado à deficiência de vitamina $\mathrm{D}$, ao passo que essa delimitação clínica aumenta a morbidade nos grupos de randomização e reduz a resposta imune à infecção por carga viral. Da mesma forma, pacientes que sofrem de Síndrome Respiratória Aguda (SDRA) acabam precisando de suporte intensivo. Quanto às delimitações geográficas, sabe-se que a escassez de raios UVB em regiões de latitudes extremas inibem a formação constante e em proporções adequadas de vitamina $\mathrm{D}$, contribuindo assim para a falta de expressividade de fatores imunológicos e mediadores químicos. Especificamente para os casos de SDRA, os fatores 
imunológicos exacerbados permitem a ampliação reacional de citocinas em cascatas (Brito et al., 2020).

Através das coletas demográficas de proporção ao longo da pandemia, foi possível notar que a maior taxa de letalidade das cepas iniciais de COVID-19, se expressou preferencialmente em segmentos populacionais acima dos 80 anos, em sua maioria com morbidades associadas, ou com difícil acesso aos recursos do sistema de saúde. Entre os grupos que se inseriram nessas perspectivas supracitadas, as variantes de vulnerabilidade social estão diretamente relacionadas ao prognóstico clínico desses quadros de infecção - nos piores casos, o nível de atenção primária e de atenção é muitas vezes negligenciado (Barbosa et al., 2020).

Em relação aos indicadores espaciais, sabe-se que em diferentes projeções da análise global, a mortalidade por infecção pelo COVID-19 está diretamente associada às desigualdades socioespaciais, principalmente em relação às taxas de desemprego e inacessibilidade diante dos processos distributivos de saúde. Também se revelam, entre as formas de disparidade social de impacto assertivo, as desigualdades étnicas, de escolaridade e consequente distribuição de renda, delimitando áreas geoespaciais responsáveis pelos maiores casos de letalidade da pandemia. Como outro fator, oculto na maioria das análises, inclui-se a poluição de partículas presentes no ar, principalmente em grandes centros urbanos e cidades asiáticas, por exemplo, determinando um índice de danos consideráveis à saúde das pessoas infectadas pelo vírus causador do coronavírus (Sun et al., 2021).

Como falha no controle do processo de isolamento, somado a deficiências persistentes ao longo das políticas públicas, o cenário nacional, no entanto, surge com um quadro preocupante no que se refere ao controle da mortalidade relacionada à pandemia, enquanto diferentes regiões brasileiras são tão distintas no cuidado na ação preventiva, que acabam inevitavelmente por determinar fluxos de casuística singulares no que diz respeito à saúde pública. Enfrentando dificuldades não apenas relacionadas ao sistema estrutural, mas também quanto à distribuição de vacinas e exames diagnósticos (Marinho et al., 2020).

Além disso, no que se refere ao espectro de pesquisa clínico-epidemiológica, sabe-se que a alta prevalência de comorbidades associadas ao quadro de infecção piora o prognóstico clínico, o tempo e a hospitalização e o risco de mortalidade especificamente na população idosa. Mediante as taxas de randomização e acurácia, pode-se observar que o maior risco de doenças oferecidas à saúde geriátrica consiste na associação da infecção SARS-CoV-2 e doenças cardiovasculares, principalmente devido à tríade de hipertensão arterial sistêmica, obesidade e diabetes mellitus, seguido por doença renal crônica e problemas respiratórios crônicos e doenças hepáticas em geral. Assim, concluiu-se que a infecção por COVID-19 é capaz de desestabilizar patologias específicas e anteriormente recorrentes, gerando maior índice de comprometimento sistêmico na maioria dos pacientes observados (Posso et al., 2020).

Não obstante, é válido pontuar que, mesmo com o surgimento de vacinas específicas, associadas a medidas de controle de saúde em massa, o primeiro trimestre de 2021 evidenciou o surgimento de novas cepas virais de COVID-19, processo vinculado às mutações de anticorpos, que assim asseguram maior resistência e potencial de disseminação dessas variantes. Mediante o aparecimento dessas novas linhagens, os estudos de randomização clínica realizados pela comunidade científica têm evidenciado a correlação entre as taxas de reinfecção por SARS-CoV-2 e a redução da imunidade protetora dos indivíduos submetidos à análise, havendo impacto e agravos em saúde nas populações predominantemente jovens (entre 20 e 40 anos), em relação à primeira fase de disseminação viral (Freitas et al., 2021).

\subsection{Vitamina D em casos severos (idosos)}

A relação entre a deficiência de vitamina D com o pior prognóstico em idosos também tem sido discutida na literatura, visto que, apesar deste grupo não estar mais propenso a contrair o vírus, tendem a apresentar complicações mais graves e ter maior taxa de mortalidade (Chen et al., 2019; Huang et al., 2020; Freitas et al., 2021;).

Em um estudo chinês multicêntrico retrospectivo, a análise regressiva multivariável determinou que a idade estava associada com o aumento de mortes hospitalares (OR 1,10, 95\% CI 1,03-1,17, por ano aumentado; $\mathrm{p}=0,0043$ ). A presença de 
comorbidades, como doenças cardiovasculares, diabetes e questões imunológicas justificam um desfecho mais negativo quando comparado aos indivíduos saudáveis (Zhou et al., 2020).

Apesar de tais fatores, a presença de comorbidades unicamente não justifica a preponderância da maior incidência de casos severos em idosos (Phelan et al., 2020). A imunosenescência, o sistema imunológico, incluindo sua capacidade de reconhecer, ativar o sistema, obliterar e remover um vírus como o SARS-CoV-2, são prejudicados de uma forma extremamente imprevisível com o aumento da idade. Evidências mostram que o processo inflamatório e a consequente liberação da tempestade de citocinas está diretamente relacionado com a obesidade, dietas nutricionalmente desequilibradas e hábitos de vida sedentários (Sanada et al., 2018). Dessa forma, a insuficiência de vitamina D está atrelada à baixa exposição à luz solar UVB, entre 290-315 $\mathrm{nm}$, à concentração de melanina na pele, esta que tem a capacidade de síntese de vitamina D diminuída com o passar dos anos (Buford et al., 2018; Griffin et al., 2020). Aliado a isto, a hipovitaminose citada também está associada à comorbidades, como doenças cardiovasculares, doença isquêmica cardíaca e morte precoce, embora a suplementação da vitamina D não mostre desfechos favoráveis na diminuição desses eventos cardiovasculares.

A infecção pela COVID-19 mostrou-se com uma incidência variada ao longo das diferentes latitudes, estando no hemisfério norte as maiores taxas de mortalidade. Isso revela a potencial relação entre baixas taxas de vitamina $\mathrm{D}$, a maior severidade dos casos da SARS-CoV-2 em idosos, consequentemente mais propensos a terem comorbidades. As taxas de mortalidade mais altas observadas em países acima de $35^{\circ} \mathrm{C}$ Norte podem ser devidas a um aumento da prevalência de pessoas mais velhas no norte da Europa, que têm maior risco de ter comorbidades cardiopulmonares e metabólicas (Panarese \& Shahini, 2020), bem como aqueles com deficiência de vitamina D, especialmente durante os meses de inverno, quando os níveis do micronutriente são mais baixos (Griffin et al., 2020; Rhodes et al., 2020). Em contrapartida, países como Brasil e México mostram-se como exceção à tal regra, o que revela a diversidade da natureza multifatorial da COVID-19.

Idosos possuem maior risco de hipovitaminose, e produzem a vitamina $\mathrm{D}$ em uma taxa $75 \%$ menor que adultos jovens, considerando uma exposição solar regular. Aliado a isso, a resistência intestinal à $1,25(\mathrm{OH})_{2} \mathrm{D}$ é aumentada com o passar dos anos, levando ao comprometimento da absorção intestinal de cálcio associada aos níveis reduzidos de receptores para a vitamina D (Phelan et al., 2020).

Curiosamente, quase 50\% de todas as mortes ocorridas devido a COVID-19 na Irlanda envolvem residentes de asilos (BBC News, 2020). Também vale a pena destacar que a estratégia de "cocooning" defendida pelo governo irlandês serve para reduzir ainda mais o acesso à luz solar UVB para aqueles em maior risco com potencial exacerbação da deficiência de vitamina D, o que piora o prognóstico dos idosos.

Metanálises recentes de ensaios clínicos randomizados concluíram que o uso de suplementos de vitamina D foi associado a menor mortalidade total em idosos, predominantemente participantes com deficiência de vitamina D (Bjelakovic et al., 2014; Keum et al., 2019). Embora seja importante suplementar com vitamina D durante os períodos de isolamento com mínima exposição ao sol, o público precisa ser aconselhado contra o uso de altas doses de vitamina $\mathrm{D}$, que podem ser prejudiciais (McKenna \& Flynn, 2020). Criticamente, os conselhos dados ao público devem ser baseados em evidências, precisos, claros, acessíveis, oportunos e alinhados às recomendações governamentais, diferente do que está sendo visto em alguns países.

\section{Conclusão}

As propriedades imunomoduladoras da vitamina D e o seu papel na regulação e na supressão de resposta de citocinas inflamatórias nas infecções respiratórias virais foram evidenciadas em estudos experimentais. Apesar das evidências, são necessários estudos prospectivos futuros para examinar se a hipovitaminose D é um dos mecanismos que promovem a rápida progressão da doença. Também foram observados importantes mecanismos moleculares pelos quais a vitamina $\mathrm{D}$ regula a resposta imune frente ao vírus SARS-CoV-2, mesmo assim, a suplementação com esta vitamina em protocolos preventivos e 
terapêuticos ainda permanecem indicados como uma estratégia adjuvante promissora que necessita de mais estudos. Aspectos genéticos podem influenciar no status da vitamina D no organismo e na escolha da dose de suplementação, sendo necessárias futuras investigações que relacionem o perfil genético e a resposta antiviral. Ainda merecem atenção os ensaios clínicos randomizados que avaliem a influência da suplementação com vitamina D no controle e progressão do quadro clínico da COVID19, especialmente nos países onde há a convocação de profissionais da saúde pública, com o apoio dos governantes, para priorizar e fortalecer as recomendações sobre a suplementação com vitamina D.

Tendo em vista o exposto, o contexto pandêmico oriundo da disseminação do COVID-19 resultou em um conjunto de ressignificações de propostas e perspectivas socioambientais outrora estabelecidas. Mediante a produção desse supracitado trabalho, espera-se que as produções vindouras permitam o estabelecimento de um aporte social e comunitário através de um olhar crítico, respaldado nas fundamentações bioquímicas e farmacológicas imprescindíveis à Ciência, à exemplo da importância de suplementação da vitamina D, referida ao longo do presente estudo. Não obstante, sugere-se, que os novos conhecimentos delimitados e correlacionados com o contexto do SARS-CoV-2, permitam um melhor direcionamento explicativo acerca das diferenças entre os processos infecciosos a qual os indivíduos são submetidos, democratizando o conhecimento no que concerne ao vínculo entre a propagação viral referida e os subtipos sanguíneos, além de outros tipos de vitaminas, compostos ou metabólitos de relevância para o combate ou redução dos quadros de infecção e propagação viral. Revela-se como fundamental, ainda assim, que os benefícios gerados pelos conhecimentos alçados pelos futuros trabalhos, delimitados também por esse panorama, possam contribuir significativamente para o desenvolvimento das mais diversas comunidades sociais e eixos técnicos, assegurando-se o respeito e o compromisso assertivo com a vida humana.

\section{Referências}

Al Mheid, I., \& Quyyumi, A. A. (2017). Vitamin D and cardiovascular disease: controversy unresolved. J Am Coll Cardiol, 70(1), 89-100.

Alexander, J., Tinkov, A., Strand, T. A., Alehagen, U., Skalny, A., \& Aaseth, J. (2020). Early nutritional interventions with zinc, selenium and vitamin D for raising anti-viral resistance against progressive COVID-19. Nutrients, 12(8), 2358.

Bae, M., \& Kim, H. (2020). The role of vitamin C, vitamin D, and selenium in immune system against COVID-19. Molecules, 25(22), 5346.

Barbosa, I. R., Galvão, M. H. R., Souza, T. A. D., Gomes, S. M., Medeiros, A. D. A., \& Lima, K. C. D. (2020). Incidência e mortalidade por COVID-19 na população idosa brasileira e sua relação com indicadores contextuais: um estudo ecológico. Rev Bras Geriat Gerontol, 23 (1), 1-11.

BBC News (2020) Coronavirus: Almost half of Irish Covid-19 deaths in care homes. Available from: https://www.bbc.com/news/world-europe-52399869.

Bertoldi G, Gianesello L, Calò L. A. Letter: ACE2, Rho kinase inhibition and the potential role of vitamin D against COVID-19. AP\&T's 2020; 52(3):577-578.

Bikle, D., Bouillon, R., Thadhani, R. \& Schoenmakers, I. (2017). Vitamin D metabolites in captivity? Should we measure free or total 25(OH)D to assess vitamin D status? J Steroid Biochem Mol Biol, 173, 105-116.

Bjelakovic, G., Gluud, L. L., Nikolova, D., Whitfield, K., Wetterslev, J., Simonetti, R. G. \& Gluud, C. (2014). Vitamin D supplementation for prevention of mortality in adults. Cochrane Database Syst Rev, 1, 1-257.

Bourhia, M., Amrati, F. E.-Z., Ullah, R., Alqahtani, A. S., Bousta, D., Ibenmoussa, S. \& Khlil, N. (2020). Coronavirus Treatments: What Drugs Might Work Against COVID-19? Nat Prod Commun, 15(7), 1-7.

Brito, D. T. M., Ribeiro, L. H. C., de Barros Silva, R., \& Hilário, C (2020). Breve relato: os benefícios da vitamina D na COVID-19. Anais da 17ª Jornada UNIFACS de Inic Cient, 1-4.

Buford, T. W., Carter, C. S., VanDerPol, W. J., Chen, D., Lefkowitz, E. J., Eipers, P., D. Morrow, C., \& Bamman, M. M. (2018). Composition and richness of the serum microbiome differ by age and link to systemic inflammation. GeroScience, 40(3), 257-268.

Carpagnano, G. E., Di Lecce, V., Quaranta, V. N., Zito, A., Buonamico, E., Capozza, E., Palumbo, A., Di Gioia, G., Valerio, V. N., \& Resta, O. (2021). Vitamin $\mathrm{D}$ deficiency as a predictor of poor prognosis in patients with acute respiratory failure due to COVID-19. J Endocrinol Investig, 44(4), 765-771.

Castro, L. C. G. (2011). O sistema endocrinológico vitamina D. Arq Bras Endocrinol Metab, 55(8), 566-575.

Chen, T., Wu, D. I., Chen, H., Yan, W., Yang, D., Chen, G., Ma, K., Xu, D., Yu, H., Wang, H., Wang, T., Guo, W., Chen, J., Ding, C., Zhang, X., Huang, J., Han, M., Li, S., Luo, X., Zhao, J. \& Ning, Q. (2020). Clinical characteristics of 113 deceased patients with coronavirus disease 2019: retrospective study. BMJ, 368.

Editorial Nature. How epidemiology has shaped the COVID pandemic. Nature 2021; 589(7843), 491-492. 
Fiske, C. T., Blackman, A., Maruri, F., Rebeiro, P. F., Huaman, M., Kator, J., Alggod, H. M. S., Sterling, T. R. (2019). Increased vitamin D receptor expression from macrophages after stimulation with M. tuberculosis among persons who have recovered from extrapulmonary tuberculosis. BMC infectious diseases, 19(1), $1-9$.

Freitas, A. R. R., Giovanetti, M., \& Alcantara, L. C. J. (2021). Variantes emergentes do SARS-CoV-2 e suas implicações na saúde coletiva. IAJMH, 4.

Gauzzi, M. C., \& Fantuzzi, L. (2020). Reply to Jakovac: COVID-19, vitamin D, and type I interferon. Am J Physiol Endocrinol Metab.

Griffin, T. P., Wall, D., Blake, L., Griffin, D. G., Robinson, S., Bell, M., Mulkerrin, E. C. \& O’Shea, P. M. (2020). Higher risk of vitamin D insufficiency/deficiency for rural than urban dwellers. J Steroid Biochem, 197, 105547.

Haan, K., Groeneveld, A. J., de Geus, H. R., Egal, M., \& Struijs, A. (2014). Vitamin D deficiency as a risk factor for infection, sepsis and mortality in the critically ill: systematic review and meta-analysis. Critical care, 18(6), 1-8.

Hermant, P., \& Michiels, T. (2014). Interferon- $\lambda$ in the context of viral infections: production, response and therapeutic implications. IEIIS, $6(5), 563-574$.

Huang, C., Wang, Y., Li, X., Ren, L., Zhao, J., Hu, Y., Zhang, L., Fan, G., Xu, J., Gu, X., Cheng, Z., Yu, T., Xia, J., Wei, Y., Wu, W., Xie, X., Yin, W., Li, H., Liu, M., Xiao, Y., Gao, H., Guo, L., Xie, J., Wang, G., Jiang, R., Gao, Z., Jin, Q., Wang, J \& Cao, B. (2020). Clinical features of patients infected with 2019 novel coronavirus in Wuhan, China. Lancet, 395(10223), 497-506.

Ilie, P. C., Stefanescu, S., \& Smith, L. (2020). The role of vitamin D in the prevention of coronavirus disease 2019 infection and mortality. Aging Clinl Exp Res, 32(7), 1195-1198.

Jean, G., Souberbielle, J. C. \& Chazot, C. (2017) Vitamin D in Chronic Kidney Disease and Dialysis Patients. Nutrients, 9(4), 328-343.

Keum, N., Lee, D. H., Greenwood, D. C., Manson, J. E., \& Giovannucci, E. (2019). Vitamin D supplementation and total cancer incidence and mortality: a meta-analysis of randomized controlled trials. Ann Oncol, 30(5), 733-743.

Khan, R. J., Jha, R. K., Singh, E., Jain, M., Amera, G. M., Singh, R. P., Jayaraman M., Singh, A. K. (2020). Identificação de candidatos promissores a drogas antivirais contra a proteína não estrutural 15 (NSP15) do SARS-CoV-2: um estudo de reaproveitamento de drogas assistido in silico. J Biomol Struct Dyn, 1 11 .

Kloc, M., Ghobrial, R. M., Lipińska-Opałka, A., Wawrzyniak, A., Zdanowski, R., Kalicki, B., \& Kubiak, J. Z. (2021). Effects of vitamin D on macrophages and myeloid-derived suppressor cells (MDSCs) hyperinflammatory response in the lungs of COVID-19 patients. Cell Immunol, 360 , 104259.

Koche, J. C. (2011). Fundamentos de Metodologia Científica. (1a ed.). Rio de Janeiro: Vozes.

Kongsbak, M., Levring, T. B., Geisler, C., \& Essen, M. R. (2013). The vitamin d receptor and T cell function. Front Immunol, 4 , 148.

Kratz, D. B., Silva, G. S. \& Tenfen, A. (2018). Deficiência de vitamina D (250H) e seu impacto na qualidade de vida: uma revisão de literatura. Revista RBAC, $50(2), 118-123$

Lichtenstein, A., Ferreira-Júnior, M., Sales, M. M., Aguiar, F. B. D., Fonseca, L. A. M., Sumita, N. M., \& Duarte, A. J. (2013). vitamina D: ações extraósseas e uso racional. $R A M B, 59,495-506$.

Liu, G., Hong, T. \& Yang, J. (2020). A Single Large Dose of Vitamin D Could be Used as a Means of Coronavirus Disease 2019 Prevention and Treatment. Drug Des Devel Ther, 14, 3429-3434.

Liu, L. C. Y., Voors, A. A., Veldhuisen, D. J., Veer, E., Belonje, A. M., Szymanski, M. K., Silljé, H. H. W., Gilst, W. H., Jaarsma, T. \& Boer, R. A. (2011). Vitamin D status and outcomes in heart failure patients. EurJHF, 13(6), 619-625.

Liu, N., Sun, J., Wang, X., Zhang, T., Zhao, M., \& Li, H. (2021). Low vitamin D status is associated with coronavirus disease 2019 outcomes: A systematic review and meta-analysis. Int J Infect Dis, 104, 58-64.

Mardani, R., Alamdary, A., Nasab, S. M, Gholami, R., Ahmadi, N., \& Gholami, A. (2020). Associação da vitamina D com a modulação da gravidade da doença em COVID-19. Virus research, $289,198148$.

Marinho, P. R. D., Cordeiro, G. M., Coelho, H. F., \& Brandão, S. C. S. (2020). Covid-19 in Brazil: A sad scenario. Cytokine Growth Factor Rev, $20,1-4$.

Martineau, A. R., \& Forouhi, N. G. (2020). Vitamin D for COVID-19: a case to answer? Lancet Diabetes Endocrinol, 8(9), 735-736.

McKenna, M. J., \& Flynn, M. A. T. (2020). Covid-19 cocooning and vitamin D intake requirements. Ir Med J, $113,79$.

Mendy, A., Apewokin, S., Wells, A. A., \& Morrow, A. L. (2020). Factors associated with hospitalization and disease severity in a racially and ethnically diverse population of COVID-19 patients. MedRxiv.

Merzon, E., Tworowski, D., Gorohovski, A., Vinker, S., Cohen, G. A., \& Green, I. Frenkel-Morgenstern M. (2020). Low plasma 25 (OH) vitamin D level is associated with increased risk of COVID-19 infection: an Israeli population-based study. FEBS Journal, 287(17), 3693-3702.

Newmark, H., Dantoft, W., \& Ghazal, P. (2017). Origem evolutiva do eixo interferon-imune metabólico: a ligação esterol-vitamina D. Front immunol, 8, 62.

Nickbakhsh, S., Mair, C., Matthews, L., Reeve, R., Johnson, P. C., Thorburn, F., Wissmann, B., Reynolds, A., McMenamin, J., Gunson, R.N. \& Murcia, P. R. (2019). Virus-virus interactions impact the population dynamics of influenza and the common cold. PNAS, 116(52), 27142-27150.

Panarese, A., \& Shahini, E. (2020). COVID-19, and vitamin D. AP\&T, 51(10), 993. 
Phelan, A. L., Katz, R., \& Gostin, L. O. (2020). The novel coronavirus originating in Wuhan, China: challenges for global health governance. Jama, 323(8), 709-710.

Posso, M., Comas, M., Román, M., Domingo, L., Louro, J., González, C., Sala, M., Anglès, A., Cirera, I., Cots, F., Frías, V., Gea, J., Güerri-Fernández, R., Masclans, J. R., Noguès, X., Vázquez, O., Villar-García, J., Horcajada, J. P., Pascual, J. \& Castells, X. (2020). Comorbidities and mortality in patients with COVID-19 aged 60 years and older in a university hospital in Spain. Arch bronconeumol., 56(11), 756.

Rhodes, J. M., Subramanian, S., Laird, E., \& Kenny, R. A. (2020). low population mortality from COVID-19 in countries south of latitude 35 degrees North supports vitamin D as a factor determining severity. Aliment Pharmacol Ther, 51(12), 1434.

Sanada, F., Taniyama, Y., Muratsu, J., Otsu, R., Shimizu, H., Rakugi, H., \& Morishita, R. (2018). Source of chronic inflammation in Aging. Fronti. Cardiovasc., 5,12 .

Shalayel, M. H., Al-Mazaideh, G. M., Aladaileh, S. H., Al-Swailmi, F. K. e Al-Thiabat, M. G. (2020). A vitamina D é um inibidor potencial de COVID-19: acoplamento molecular in silico ao local de ligação da endoribonuclease Nsp15 da SARS-CoV-2. Pak J Pharm Sci, 33 (5).

Siuka, D., Pfeifer, M., \& Pinter, B. (2020). Vitamin D supplementation during the COVID-19 pandemic. Mayo Clin Proc, 95(8), 1804-1805.

Sousa, S. M. (2016). A vitamina D e o seu papel na prevenção de doenças. Tese de mestrado, Universidade Fernando Pessoa, Porto, Portugal.

Sun, Y., Hu, X., \& Xie, J. (2021). Spatial inequalities of COVID-19 mortality rate in relation to socioeconomic and environmental factors across England. Sci Total Environ, 758, 143595, 1-11.

Teixeira, H. C. L., Dias, L. S., Bizarro, H. D. S. \& Castro, J. M. A. (2018). Efeitos contrastantes da vitamina D sobre a resposta imune inata e adquirida e seu impacto na recuperação da tuberculose. HU Revista, 44(3), 369-378.

Zhou, F., Yu, T., Du, R., Fan, G., Liu, Y., Liu, Z., Xiang J., Wang Y., Song B., Xiaoying G., Guan L., Wey Y., Li H., Wu X., Xu J., Tu S., Zhang Y., Chen H., \& Cao, B. (2020). Clinical course and risk factors for mortality of adult inpatients with COVID-19 in Wuhan, China: a retrospective cohort study. Lancet, 395(10229), 1054-1062. 